\title{
Mechanical Behavior of Zr-Based Bulk Metallic Glasses
}

\author{
S. Nowak, ${ }^{1, \mathrm{a}}$ P. Ochin, ${ }^{1, \mathrm{~b}}$ A. Pasko, ${ }^{1}$ S. Guérin, ${ }^{1}$ and Y. Champion ${ }^{1, \mathrm{c}}$ \\ ${ }^{1}$ ICMPE-CNRS UMR7182, Université Paris, Vitry-sur-Seine, France \\ a nowak@icmpe.cnrs.fr, ${ }^{\mathrm{b}}$ ochin@icmpe.cnrs.fr, ${ }^{\mathrm{c}}$ champion@icmpe.cnrs.fr
}

Bulk metallic glasses have a very high corrosion resistance and mechanical strength. Bulk metallic glasses show elastic-perfectly plastic behavior with an extended region of elastic strain $(\approx 2 \%)$. But at room temperature their macroscopic plasticity is weak even though a local plastic strain is observed in shear bands. A relaxation analysis allowed studying micro-mechanisms of plastic deformation and estimating the apparent activation volume $\left(\approx 2000 A^{3}\right)$.

Keywords: bulk metallic glasses, compression test, stress relaxation, mechanical properties.

Introduction. Amorphous metallic alloys also called metallic glasses are characterized by absence of atomic long-range order. Bulk metallic glasses (BMG) exhibit a very high strength $(\approx 1,6 \mathrm{GPa})$ and elasticity $(\approx 2 \%)$. However, at room temperature, they have low ductility because of the localization of the plastic strain which is concentrated in a few thin shear bands. Deformation behavior of BMGs is completely different than crystallized metals (no dislocation). Spaepen [1] and Argon [2] describe the deformation as the result of jumps of respectively single-atom or group of atoms in "holes" (free volumes) large enough.

Zr-based BMG have a high GFA (Glass Forming Ability) and particularly the alloy $\mathrm{Zr}_{57} \mathrm{Cu}_{20} \mathrm{Al}_{10} \mathrm{Ti}_{8} \mathrm{Ni}_{5}$ which is the studied alloy in this paper.

BMG Synthesis and Characterization. Initially, the five pure elements are melted by electromagnetic induction heating in a water-cooled copper crucible under $\mathrm{He}$ atmosphere (Fig. 1a). From 20 to $35 \mathrm{~g}$ of BMGs are obtained by re-melting using electromagnetic levitation under He atmosphere and casting into a copper mold (Fig. 1b).

Different shapes of samples are produced, depending on the subsequent use: $20 \times 35 \times 5 \mathrm{~mm}$ sheets for compression tests, rods with $10 \mathrm{~mm}$ diameter for transmission electron microscopy analysis and wedge shaped samples for the evaluation of the glass forming ability (GFA).

For compression tests, rectangular shaped samples, $4 \times 4 \mathrm{~mm}$ of cross-sectional area and $6 \mathrm{~mm}$ height, were machined and then polished.

$\mathrm{X}$-ray diffraction and TEM analysis were carried out to control the amorphous state of the as-cast samples (presence of broad diffuse peaks for XRD, and diffuse rings for TEM).

The glass transition temperature $\left(T_{g}=660 \mathrm{~K}\right)$ and the crystallization temperature $\left(T_{x}=719 \mathrm{~K}\right)$ of the alloy were measured using differential scanning calorimeter (DSC) and the liquidus temperature $\left(T_{l}=1156 \mathrm{~K}\right)$ was measured using DTA. Heating rate of $20 \mathrm{~K} / \mathrm{min}$ was applied for each analysis.

Mechanical Behavior. Uniaxial compression test under quasi-static loading at room temperature was performed. BMG exhibits a perfect elastic deformation behavior followed by a catastrophic brittle fracture with no yielding (Fig. 2). The fracture stress is $1634 \mathrm{MPa}$ and the region of elastic strain is extended $(\approx 2 \%)$. Though macroscopic plasticity is low, local plastic strain is observed in shear bands (Fig. 3).

Typical morphology of the fracture surface of a BMG, at room-temperature in compression, is shown in Fig. 4. Veins with liquid droplets were observed in the entire fracture surface. It was demonstrated that shear localization induces a temperature rise (more than $900^{\circ} \mathrm{C}$ at the final-fracture moment, i.e., higher than $T_{l}$ ) and that deformation is then related to a local decrease of the viscosity in the shear bands [3]. 


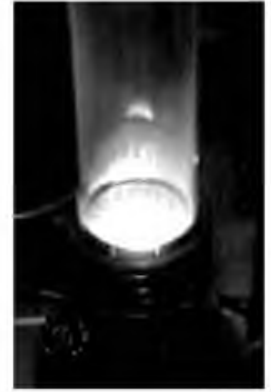

a

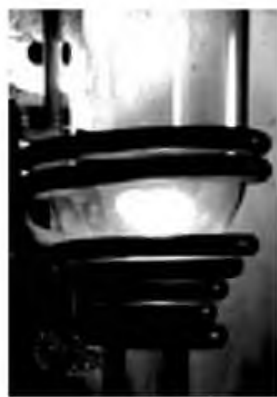

b

Fig. 1

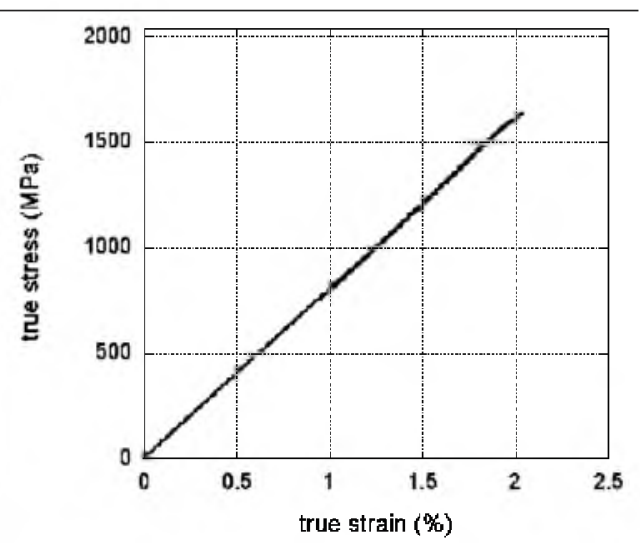

Fig. 2

Fig. 1. (a) water-cooled copper crucible (b) electromagnetic levitation.

Fig. 2. Stress-strain curve of the $\mathrm{Zr}_{57} \mathrm{Cu}_{20} \mathrm{Al}_{10} \mathrm{Ni}_{8} \mathrm{Ti}_{5} \mathrm{BMG}$ deformed at room temperature at a strain rate of $2 \cdot 10^{-5} \mathrm{~s}^{-1}$.

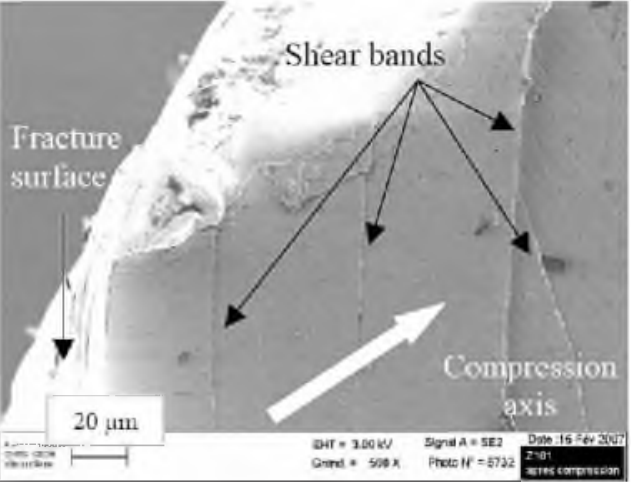

Fig. 3

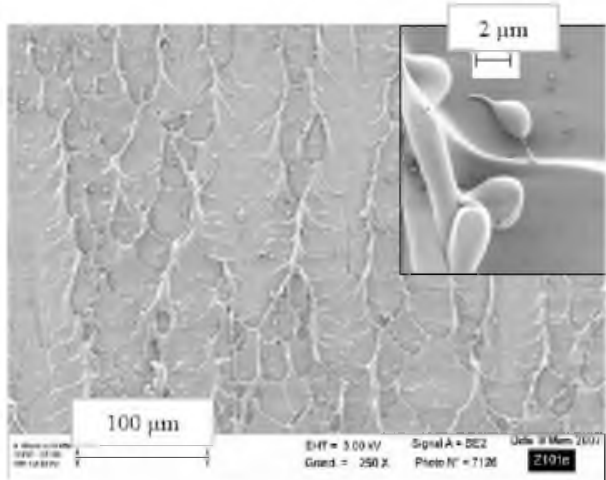

Fig. 4

Fig. 3. View of free surface, parallel to the compression direction, with visible shear bands. Shear band thickness is about $20 \mathrm{~nm}$ [5].

Fig. 4. Fracture surface with veins and liquid droplets (insert)

The fracture angle was measured for two samples: one was $41^{\circ}$ (Fig. 5), the other $45^{\circ}$. These values indicate that BMG follows the Mohr-Coulomb criterion for plastic yielding in compression. This behavior is observed for many $\mathrm{BMG}$, such as $\mathrm{Zr}_{574} \mathrm{Cu}_{16}{ }_{4} \mathrm{Ni}_{82} \mathrm{Al}_{10}$ [4].

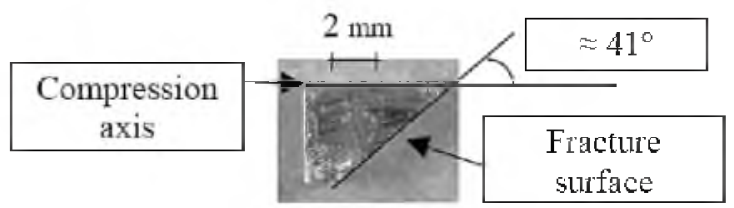

Fig. 5. Fractured sample.

Stress Relaxation Analysis. A relaxation test was performed at room temperature to approach the micromechanisms of deformation. In literature, most experiments were conducted at temperatures close to $T_{g}[1,6]$, BMG having homogeneous deformation at these temperatures. In our experiment, an attempt is made to examine the localized 
deformation in shear bands. Relaxation is a method allowing the measurement of the rheologic and the mechanistic parameters without failure of the sample and at a macroscopic scale (in contrast to the nano-indentation investigating confine plasticity).

The sample is loaded with a strain rate of $\dot{\varepsilon}=5 \cdot 10^{-5} \mathrm{~s}^{-1}$. The displacement of the cross head of the testing machine is stopped just before the catastrophic failure of the sample. The total deformation is remained constant until the end of the experiment $(\approx 160,000 \mathrm{~s})$. Consequently, since total deformation is the result of plastic and elastic deformation:

$$
\dot{\varepsilon}_{\text {plastic }}=-\dot{\varepsilon}_{\text {elastic }} .
$$

The shear stress variation as a function of time is plotted in Fig. 6. Three domains are defined to describe the curve. Between $300 \mathrm{~s}$ (onset of the relaxation) and $8000 \mathrm{~s}$, the stress decreases slowly $\left(\Delta \tau_{\max }=7 \mathrm{MPa}\right.$ ) following the classical logarithmic relation. Then, after a transitory plateau, the curve globally increases until 100,000 s and finally stabilizes in the third part.

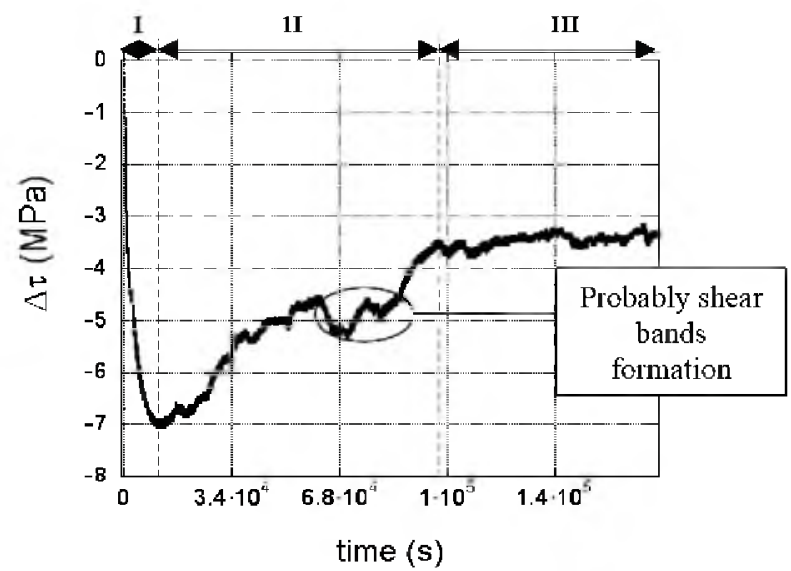

Fig. 6. Plot of the shear stress variation as a function of time.

I. The first domain follows the logarithmic function [7]

$$
\Delta \tau=\tau-\tau_{0}=-\frac{k T}{V_{s p p}} \ln \left(1+\frac{t}{C}\right),
$$

where $\tau$ is applied shear stress, $\tau_{0}$ is applied shear stress at the beginning of the relaxation, $t$ is time, $V_{a p p}$ is apparent activation volume, $C$ is time factor, $k$ is Boltzmann constant, and $T$ absolute temperature. $V_{a p p}$ is the atomic volume involved in an elementary thermally activated event. At the onset of the relaxation, the slope is almost infinite and $V_{a p p}$ equal to zero. Then the curve can be perfectly fitted between 1000 and $3500 \mathrm{~s}$ by the logarithmic relation and the activation volume $V_{a p p}$ is estimated to $2000 \AA^{3}$ (corresponding to $150 \Omega$, where $\Omega$ is the average atomic volume), which is reasonable compared to high temperature measurement [8].

II. An increase of $\tau$ is observed, which is probably related to an energy release. Such behavior is rather unusual. It was verified that it was not in relation with experiment artifact: stress variations induced by the machine were measured as negligible compared with the sample relaxation. Moreover, experiments are performed in a room with constant temperature and the system (sample-machine) dilatation cannot be taken into account to explain the phenomenon. 
So the change between domain $I$ and $I I$ could be related to the variations in micro-structure which is most likely a crystallization in shear bands [9]. At $T>T_{g}$, Nieh et al. [10] consider amorphous phase as a Newtonian fluid and nanocrystalline particles as having a superplastic behavior. The plastic deformation strain rate is consequently expressed by

$$
\dot{\gamma}_{\text {plastic }}=\left(1-f_{v}\right) \dot{\gamma}_{\text {am }}+f_{v} \dot{\gamma}_{\text {cryst }}=\left(1-f_{v}\right) A \tau+f_{v} B \tau^{2}
$$

where $f_{v}$ is volume fraction of the crystalline phase, $A$ and $B$ are material constants, $\dot{\gamma}_{a m}$ and $\dot{\gamma}_{c r y s t}$ strain rates caused by the amorphous and the crystalline phase, respectively, and $\tau$ the applied flow stress.

Though experiment is carried out at room temperature, deformation occurring in shear bands where temperature rises should be described consistently by Eq. (3). Consequently, the plastic deformation induces a decrease of the applied stress. Nevertheless the microstructure variation could be at the origin of an internal stress release. The measured stress which increases globally would be the sum of the internal stress and the applied stress.

III. Finally, the stress reaches a value threshold, meaning no longer plastic deformation.

Conclusions. BMGs are produced by rapid cooling of a metallic alloy, avoiding atomic long-range order. That gives specific properties to the material like no ductility because of the localization of the plastic strain in shear bands. Stress relaxation allowed estimating an apparent activation volume associated to a plastic deformation and observing an evolution of deformation mode involving most likely a partial crystallization phenomenon.

Acknowledgments. This work was supported by the DGA within the framework of a "Recherche Exploratoire et Innovation" (REI No. 05C0145) under the contract No. 0634030004707565 for the PHD of one of the authors (SN). The authors are also grateful to J. L. Bonnentien, A. Valette, and M.-F. Trichet for technical support.

1. F. Spaepen, Acta Metall., 25, 407 (1977).

2. A. S. Argon, Acta Metall., 27, 47 (1979).

3. B. Yang, P. K. Liaw, G. Wang, et al., Intermetallics, 12, 1265 (2004).

4. R. T. Ott, F. Sanchez, T. Jiao, et al., Metall. Mater. Trans., 37A, 3251 (2006).

5. A. L. G. Y. Zhang, Appl. Phys. Lett., 89, 071907-1 (2006).

6. O. P. Bobrov, V. A. Khonik, K. Kitagawa, and S. N. Laptev, J. Non-Crystalline Solids, 342. 152 (2004).

7. J. Bonneville, P. Späig, J.-L. Martin, Proc. M.R.S. Symp., 364, 369 (1995).

8. M. Bletry, P. Guyot, Y. Brechet, et al., Intermetallics, 12, 1051 (2004).

9. W. H. Jiang, F. E. Pinkerton, and M. Atzmon, Scripta Mater, 48, 1195 (2003).

10. T. G. Nieh, T. Mukai, C. T. Liu, and J. Wadsworth, Scripta Mater., 40, 1021 (1999).

Received 28. 06. 2007 